\title{
Endoscopic Management of a Large Calculus in a Transplant Kidney
}

\author{
Saman Moazami ${ }^{1}$, Ilse Daehn², Lanna Cheuck ${ }^{1 *}$ \\ ${ }^{1}$ Department of Urology, Montefiore-The University Hospital, Albert Einstein College of Medicine. New York. \\ ${ }^{2}$ Division of Nephrology, Department of Medicine and The Charles Bronfman Institute for Personalized Medicine, Icahn School of Medicine at Mount
} Sinai. New York.

Received: November 3, 2016; Accepted: February 15, 2017; Published: March 18, 2017

*Corresponding author: Lanna Cheuck, DO Director of Endourology, Assistant Professor Department of Urology Montefiore-The University Hospital Albert Einstein College of Medicine 2300 Westchester Ave, Bronx, NY 10462, Tel: 718-920-4531; Fax: 929-263-3952; Email: Icheuck@ montefiore.org

\section{Introduction}

Urolithiasis is a rare condition affecting $0.4-1 \%$ of all renal transplants [1]. Stone formation in transplant patients is multifactorial, including factors such as recipient increased urine concentration, increased uric acid/calcium excretion, hyperparathyroidism, ureteral obstruction, urinary stasis, foreign bodies such as retained sutures, vesicoureteral reflux, metabolic diseases, and preexisting stone in a donor kidney [2,3]. Percutaneous Nephrolithotomy (PCNL) is the recommended treatment for stones $>1.5 \mathrm{~cm}$ in size, but carries a greater risk of morbidity when compared to endoscopic interventions [4]. We report the case of a 68-year-old male who developed a 3.5 $\mathrm{cm}$ staghorn calculus in the donor kidney one year following transplantation, and underwent successful management with staged ureteroscopic stone extraction.

\section{Case Presentation}

A 68-year-old male with a history of hypertension, morbid obesity status post gastric sleeve, type II diabetes, and chronic kidney disease underwent a right lower quadrant living, unrelated renal transplant with Simulect induction in April 2015. His immunosuppressive regimen consisted of Prednisone, Tacrolimus, and Mycophenolate.

He was referred to urology in April 2016 for gross hematuria work-up, revealing the presence of a $3.5 \mathrm{~cm}$ staghorn calculus measuring 529 Hounsfield units, with moderate hydronephrosis within the transplant kidney (Figure 1).

He was subsequently taken to the operating room for planned ureteroscopy with laser lithotripsy, and possible percutaneous nephrolithotomy. Initially, a cystogram was performed in the operating room revealing no evidence of reflux into the transplant kidney. Due to the location of the neo-orifice on the posterior right lateral wall, an angled Berenstein catheter was used to pass a wire into the renal pelvis. A second wire was passed into the collecting system using a dual lumen catheter. Due to difficulty advancing an access sheath into the transplant ureter, a Storz Flex-X2 fiber optic flexible ureteroscope was used to access the collecting system over one of the wires. A 365-micron holmium laser fiber was used to fragment the stone into small pieces. The largest stone fragments were extracted using a basket, however due to the lack of an access sheath to assist in passing the ureteroscope in and out of the renal collecting system, the decision was made to insert a ureteral stent with plan for a second stage ureteroscopy and lithotripsy.

The patient was discharged on post-operative day two, but returned to the emergency room on day four with oliguria and acute kidney injury. Non-contrast CT scan revealed an adequately placed ureteral stent with evidence of steinstrasse or "cobble stone appearance" in the proximal ureter (Figure 2). He was taken to the operating room for second look ureteroscopy and noted to have multiple soft stone fragments within the

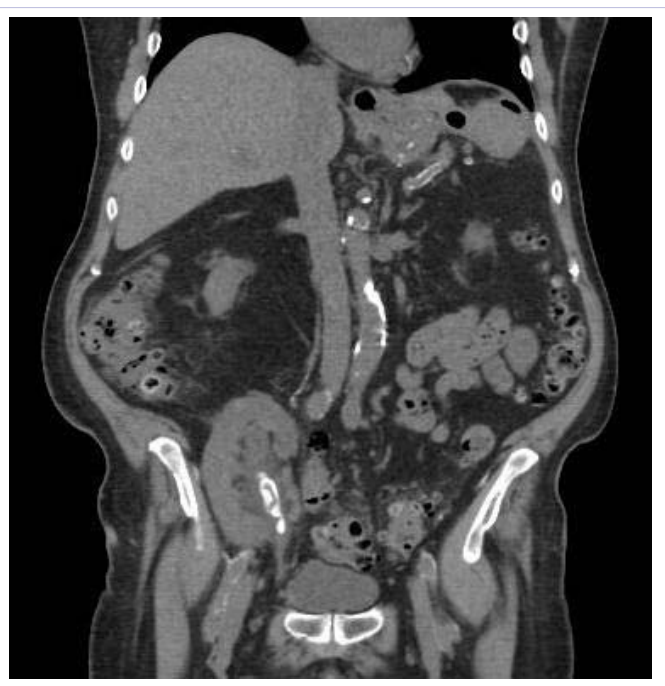

Figure 1: Initial CT scan done during hematuria evaluation, revealing a large stone approximately $3.5 \mathrm{~cm}$ in size within the transplant graft. 
proximal ureter. All clinically significant fragments were lasered, extracted, and a ureteral stent was placed. Post-operatively his creatinine improved to baseline. Stone analysis revealed calcium oxalate Calcium Oxalate Monohydrate (Whewellite) 60\% and Calcium Oxalate Dihydrate (Weddellite) $40 \%$. CT scan was done one-month post-procedure after routine removal of his stent using office cystoscopy, revealing a significant reduction in his stone burden (Figure 3). Imaging revealed two small fragments in the proximal ureter, but the patient was asymptomatic and opted for conservative management. He subsequently passed multiple fragments in his urine. Lab studies at three months post-op revealed a Cr of 1.3 and GFR 55. 24 hour urine studies demonstrated low urine volume of $1.23 \mathrm{~L} /$ day, low urine citrate of $114 \mathrm{mg}$ /day, low urine calcium (15 mg/day), borderline hyperoxaluria (40 mg/day), low $\mathrm{pH} 4.914$, and increased supersaturation of uric acid. Dietary counseling was initiated, and the patient was started on potassium citrate supplement.

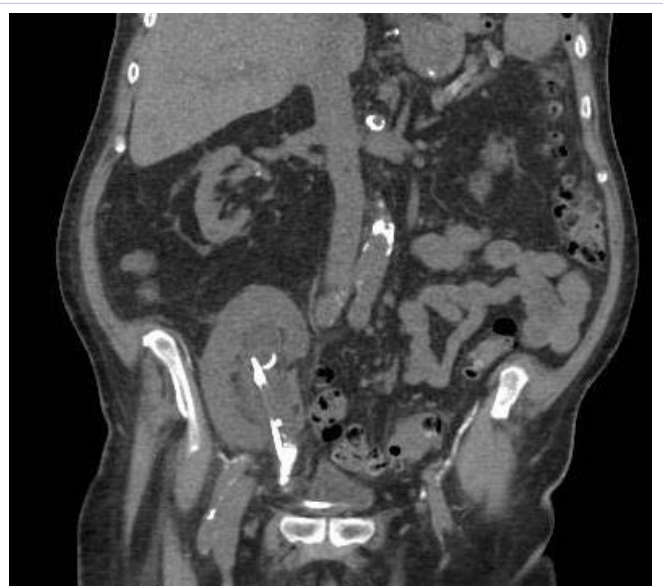

Figure 2: CT scan after the initial ureteroscopy with the presence of steinstrasse in the proximal ureter, and urinary obstruction despite placement of a ureteral stent.

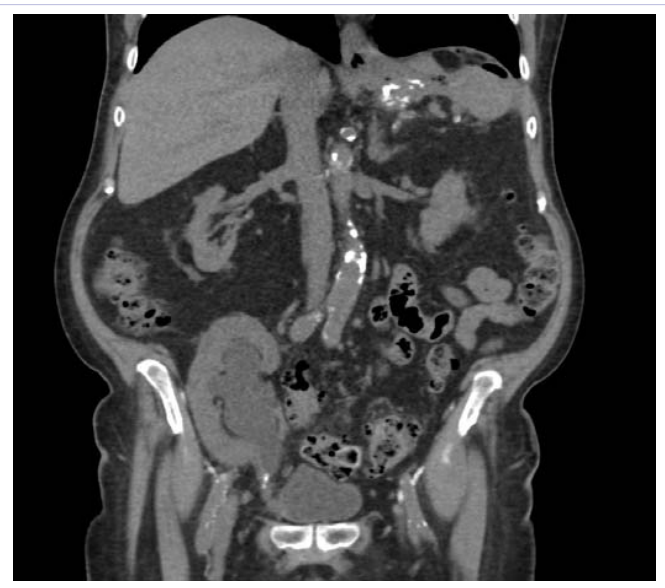

Figure 3: CT scan after the second ureteroscopy showing significant reduction in stone burden, and presence of small fragments in the proximal ureter.

\section{Discussion}

Patients with urolithiasis of a transplant graft often do not present with symptoms of colic, due to denervation of the allograft kidney and ureter [5]. Similar to our patient, nephrolithiasis is frequently a finding on evaluation of infection, hematuria, decreased urine output, or acute kidney injury. In a series of 19 patients, Klingler et al report $47 \%$ had stones that were transplanted with the allograft. As a result, they recommend routine intraoperative ultrasound at the time of transplantation [6]. For patients with de novo formation of calculi, recurrent infections, hyperparathyroidism, ureteral obstruction, urinary stasis, foreign body nidus, and metabolic disease are all potential predisposing factors $[2,7]$. While most of these patients produce calcium oxalate stones, immunosuppression with cyclosporine results in hyperuricosuria and possible susceptibility to uric acid stone formation [6].

Due to the complications associated with performing an open pyelolithotomy in immunosuppressed patients, minimally invasive options are a suitable alternative [4]. Extracorporeal shockwave lithotripsy is possible for stones less than $1.5 \mathrm{~cm}$ in size; however re-intervention rates of $61 \%$ have been reported [4]. Additionally, this approach places the patient at risk for steinstrasse in a solitary kidney. Percutaneous removal of calculi from a transplant kidney was first described in 1985 by Hulbert et al [8]. One of the largest series to date reports high success rates, with few complications. Nevertheless, there are many challenging aspects which can result in increased morbidity. Due to the invasive nature of a percutaneous approach, major and minor complications can occur in up to $7 \%$ and $25 \%$ of patients respectively, and include sepsis, adjacent organ injury, and perforation of the renal pelvis and ureter. Transfusion rate of up to $10 \%$ have been reported. $0.5 \%$ of patients develop arteriovenous fistula or pseudoaneurysm requiring embolization [9]. In traditional PCNL of a native kidney, access is obtained into a posterior calyx. In transplant kidneys, the change in renal axis requires anterior access, which may increase the risk of bleeding. Additional shortcomings of this procedure include difficulty obtaining retrograde opacification of the collecting system during fluoroscopy, as well as problems associated with tract dilation due to surrounding scar tissue. Despite the potential complications and difficulties in performing PCNL, Rifaioglu et al. had no complications in their small series of 15 patients [7]. Nevertheless, the potential risk of an invasive percutaneous approach makes endoscopic management an attractive option if technically feasible.

Endoscopic management is known to be technically challenging due to the anterior and superior location of the anastomosis near the dome of the bladder, albeit, high success rates with low incidence of complications have been reported [10]. Nevertheless, published cases describing ureteroscopic management of large stone burden within a renal allograft are limited. Our experience demonstrates that endoscopic management is a safe alternative to percutaneous nephrolithotomy, in patients with stone burden greater than 1.5 $\mathrm{cm}$ in a renal transplant graft. Although multiple interventions 
may be required, the decreased risk of ureteroscopic intervention compared to PCNL suggests this modality should be initially attempted in these patients.

\section{References}

1. Rhee BK, Bretan PN Jr, Stoller ML. Urolithiasis in renal and combined pancreas/renal transplant recipients. J Urol. 1999;161(5):1458-1462.

2. Harper JM, Samuell CT, Hallson PC, Wood SM, Mansell MA. Risk factors for calculus formation in patients with renal transplants. Br J Urol. 1994;74(2):147-150.

3. Van Gansbeke D, Zalcman M, Matos C, Simon J, Kinnaert P, Struyven J. Lithiasic complications of renal transplantation: the donor graft lithiasis concept. Urol Radiol. 1985;7(3):157-160.

4. Challacombe B, Dasgupta P, Tiptaft R, Glass J, Koffman G, Goldsmith D, et al. Multimodal management of urolithiasis in renal transplantation. BJU Int. 2005;96(3):385-389.

5. Markic D, Krpina K, Ahel J, Grskovic A, Spanjol J, Rubinic N, et al Treatment of Kidney Stone in a Kidney-Transplanted Patient with
Mini-Percutaneous Laser Lithotripsy: A Case Report. Case Rep Nephrol Dial. 2016;6(1):26-31. doi: 10.1159/000444251

6. Klingler HC, Kramer G, Lodde M, Marberger M. Urolithiasis in allograft kidneys. Urology. 2002;59(3):344-348.

7. Rifaioglu MM, Berger AD, Pengune W, Stoller ML. Percutaneous management of stones in transplanted kidneys. Urology. 2008;72(3):508-512. doi: 10.1016/j.urology.2008.05.040

8. Hulbert JC, Reddy P, Young AT, Hunter DW, Castaneda-Zuniga W, Amplatz K, et al. The percutaneous removal of calculi from transplanted kidneys. J Urol. 1985;134(2):324-326.

9. Matlaga BR, Lingeman JE. Surgical management of upper urinary tract calculi. In: Wein AJ, Kavoussi LR, Novick AC, et al, editors. Campbell-Walsh Urology. 10th edition. Chapter 48.Philadelphia: Saunders.2011;1357-1410.

10. Del Pizzo JJ, Jacobs SC, Sklar GN. Ureteroscopic evaluation in renal transplant recipients. J Endourol. 1998;12(2):135-138. doi: 10.1089/ end.1998.12.135 